\title{
A Tangible User Interface to Facilitate Learning of Trigonometry
}

\author{
https://doi.org/10.3991/ijet.v14i23.11433 \\ Francisco Zamorano Urrutia $\left({ }^{\varpi}\right)$, Catalina Cortés Loyola, Mauricio Herrera Marín \\ Universidad del Desarrollo, Santiago, Chile \\ franciscozamorano@udd.cl
}

\begin{abstract}
In mathematics education, studies reveal difficulties in the teaching-learning of trigonometry in secondary and higher education, due to the fact that students are not encouraged to achieve a deep conceptual understanding of abstract concepts. Several studies demonstrate that incorporating digital technologies has a positive impact on students' learning. However, most of the existing technologies do not consider the use of the body and multiple senses. Tangible User Interfaces (TUIs) in contrast, can host bodily interactions that have the potential of enhancing learning. Nonetheless, there is a lack of applications of TUIs for trigonometry education. This study consisted of designing and validating a tangible interface for the teaching-learning of basic concepts of trigonometry. The interface hosts a pedagogical experience that privileges exploration through physical manipulation and fosters intuitive and collaborative learning. A Pre-Test was applied to 121 students to determine previous knowledge, yielding a $29.1 \%$ performance. After two sessions using the interface, the results of a Post-Test reveal an increase of $37.1 \%$, confirming the educational effectiveness of the interface and the pedagogical experience to facilitate learning of basic concepts of trigonometry.
\end{abstract}

Keywords—design, collaborative learning, Tangible User Interfaces, interaction, trigonometry

\section{Introduction}

Trigonometry is a fundamental requirement for the study and practice of both advanced mathematics and science. In physics, for instance, periodic trigonometric functions enable the investigation of real-world phenomena [1]. In project-based disciplines such as Design, Engineering, and Architecture, trigonometry is used to design a wide range of structures, such as bridges, furniture, and bicycles. From a cognitive standpoint, trigonometry is fertile in connecting algebraic, geometric, and graphic reasoning [2]. But despite its relevance, learning and understanding trigonometry has proven to be challenging. Research shows that in secondary and higher-level education, trigonometry remains a difficult subject for both students and teachers [2-4]. To some extent, the flaws in trigonometry comprehension stem from traditional teaching practices inspired by behaviorism, where students are subjected to repetitive proce- 
dures and cyclical evaluations [5]. This approach does not usually stimulate creativity or incorporate the premise that human beings add meanings to the thought process [6]. In consequence, students resort to memorization when facing trigonometric problems [7], excluding in-depth conceptual understanding and the ability to comprehend the same concept represented in different ways [2].

Constructivism, in contrast, states that students learn by constructing something with their prior knowledge [8] in combination with newly input information to produce significant learning [9]. In this approach, students are challenged with a problem and encouraged to work on their own to solve it, thus promoting higher levels of thinking. Constructivism in education has gained traction in the last twenty years, involving the incorporation of new, more participatory methodologies and technologies into the classroom. In mathematics education, traditional teaching methods and classroom settings have been disrupted by the integration of Information and Communication Technologies (ICTs), particularly by the integration of Dynamic Mathematical Software as learning tools.

One of the most widely used tools is Geogebra, which features simultaneous geometric, synthetic, algebraic, and analytic representations of a mathematical object [10]. These objects can be explored interactively, enabling students to acquire comprehensive knowledge of a mathematical concept [11]. Geogebra supports an intuitive "learning by doing" approach [12], ultimately making the learning process more engaging [13]. Nevertheless, software-based tools such as Geogebra are usually constrained to single-user operation where the physical interaction is reduced to individual use of a mouse, not taking into consideration that human beings are able to learn collaboratively through the use of their bodies.

The theory of Embodied Cognition states that human beings internalize abstract concepts in concrete terms using ideas and modes of reasoning based on the sensorimotor system, making conceptual understanding crucially dependent on the nature of bodies and the physical environment in which they function [14, 15]. In contrast to purely digital tools, Tangible User interfaces (TUIs) enable bodily experiences that promote Embodied Cognition principles. By providing physical, manipulative inputs to control interactive systems, TUIs benefit from the haptic resources that we develop when manipulating everyday objects [16]. Although TUIs have gained popularity in education-especially in primary education-, in the teaching-learning of trigonometry there is an unexplored potential in the use of tangibility to enable a more profound understanding of abstract mathematical concepts. This gap presents an interesting opportunity to build upon the advantages of ICTs and at the same time, harvest the benefits of Embodied Cognition to learn trigonometry.

In this article, we present the learning assessment results of the design of a collaborative pedagogical experience and a TUI to facilitate the learning of basic concepts of trigonometry. By enabling exploration of abstract mathematical concepts through physical elements, the experience builds on embodied cognition to produce significative learning. Both the physical attributes of the interface and the progression of the pedagogical experience enable novices to integrate knowledge smoothly and intuitively, using multiple senses, and encouraging collaborative learning through social interaction. 


\section{Tangible User Interfaces and Embodied Cognition}

TUIs are human-computer interfaces that give physical form to digital elements, enabling the user to directly manipulate digital information with their hands [17]. The first approaches to TUIs emerged at the end of the nineties, thanks to the seminal work of Iroshi Ishii and Brigg Ullmer in the Tangible Media Group of MIT. Since then, TUIs have become more accessible and its fields of application have diversified into both the public and private sphere: kiosks in science museums, pieces of interactive art, collaborative tables in workspaces, and more recently in wearable interfaces.

A body of literature reflects on the multiple benefits offered by TUIs for education $[18,19]$, supported by the notion that hands-on activities and direct manipulation is beneficial for learning [20]. Additionally, TUIs can support synchronous co-located collaboration, which enables opportunities for both individual and collective discovery [21]. Moreover, TUIs influence the thought processes by fostering exploratory and playful behaviors that enable users to formulate hypotheses and rapidly evaluate diverse alternatives [18, 22, 23].

The embodied approach of TUIs to learning draws upon from principles of the Embodied Cognition theory, which states that the way in which we internalize new abstract concepts is by resorting to the knowledge we have acquired through corporal experiences since childhood [15]. Conceptual understanding is achieved through a mechanism called Conceptual Metaphors, that consists of a transfer-or mapping-of a source concept to a target concept. The source concepts are often concrete and have some kind of "body base", while the target concepts are often abstract and cannot be directly experienced $[6,23-25]$. Due to their manipulative nature, TUIs have the potential of concretizing abstract mathematical concepts by engaging users' transference of conceptual metaphors.

\section{Opportunity and Research Proposal}

This study was conducted at Universidad del Desarrollo, Chile, and involved a collaboration between the Design School and the Engineering School. One of the starting points of the study were the results of the Diagnostic Test that is applied to first-year Engineering students at the beginning of their first semester. For several years, results have shown that trigonometry is a subject that presents comparative deficiencies. In the 2017 Diagnostic Test, trigonometry presented the lowest performances (14\% and $17 \%$ ) within all the addressed subjects (mean performance of $28 \%$ ). These results encouraged the research team to inquire about the recurrence of these difficulties in other careers. At the Design School, for instance, there is no formal instruction on trigonometry (or any mathematics) during their course studies. It could then be assumed that their background knowledge of trigonometry would be similarly low. Since there's no diagnostic test in the Design School curriculum, the low performance hypothesis needed further assessment. Including students from both schools offered an interesting group of subjects for the study: On one hand we had Engineering students that would be exposed to formal instruction in trigonometry but presented a low 
performance at the beginning of their studies; On the other hand we had Design students that would apply trigonometry concepts intuitively in their practice but without having an actual course dedicated to mathematics instruction.

Taking into account that the literature evidences that the incorporation of ICTs into the classroom and the use of Dynamic Mathematics Software for mathematics education is beneficial for learning [10,12, 13, 27], there's a limited application of TUIs in more abstract domains such as trigonometry [28]. These findings led to consider an intervention in the learning process of undergraduate students, where the influence of a TUI could be beneficial to facilitate learning of trigonometry. As a starting point for our research, we elaborated on the following research questions: How can we learn trigonometry in a more intuitive and approachable way? Can a TUI facilitate the learning of basic concepts of trigonometry?

This study consisted of designing and validating a TUI for the teaching-learning of basic concepts of trigonometry. The interface hosts a pedagogical experience that privileges exploration, the use of intuition, and fosters collaborative learning. In this article, we report about the resulting design of the interface and the learning assessment of students exposed to its use.

\section{$4 \quad$ Application of a TUI for Trigonometry Learning}

\subsection{Description of the interface}

The proposed design is a TUI that acts as a mediator during a pedagogical experience. An instructor, along with a group of students, use the interface to explore diverse trigonometry concepts. As seen in Figure 1, the interface features tangible controllers to control digital elements on a graphical user interface (GUI) displayed on an LCD screen. The controllers are operated using several gestures (sliding, pressing, rotating) and are distributed into two zones with distinct purposes. The instructor stands on the upper side of the screen, where a series of controls enable him to navigate through the content, toggle the visualization of information, and turn sound on and off; Students stand on the lower end of the screen, where they can manipulate elements and parameters of the GUI and navigate through different sections of the sequence.

The Unit Circle was chosen as the model that sustains the exploration and learning of trigonometric concepts with the interface. The Unit Circle is a mathematical model where trigonometric functions are plotted based on a circle with radius one, centered at the origin $(0,0)$ in a cartesian plane. It is widely used in trigonometry [29] because it simplifies the calculation of sine and cosine based on an angle and helps to better understand periodic functions [30]. The interface features a rotational control that we call "Rotary Wheel", a thirty-two-centimeter ring that can be turned in 360 degrees. Rotation changes the angle values of the unit circle on screen, consequently modifying trigonometric values (e.g., value of cosine). Additional relevant information is also graphed on screen: sine and cosine values, the current angle, the cartesian plane, the sine and cosine waves, and textual and numerical values. 
The interface considers the learning benefits of integrating multiple peripheral senses (vision, touch, and sound) as a teaching-learning strategy [31]. Sound has two roles: First, to highlight relevant information. For instance, when a special angle $(0$, $30,45,60$ or 90 degrees, etc.) is reached by turning the Rotary Wheel a beep sound is triggered, indicating the user that there's something about that value that is worth exploring and analyzing; The second role is to represent trigonometric concepts as sound (e.g., sine waves represented as a variable tone to understand frequency).

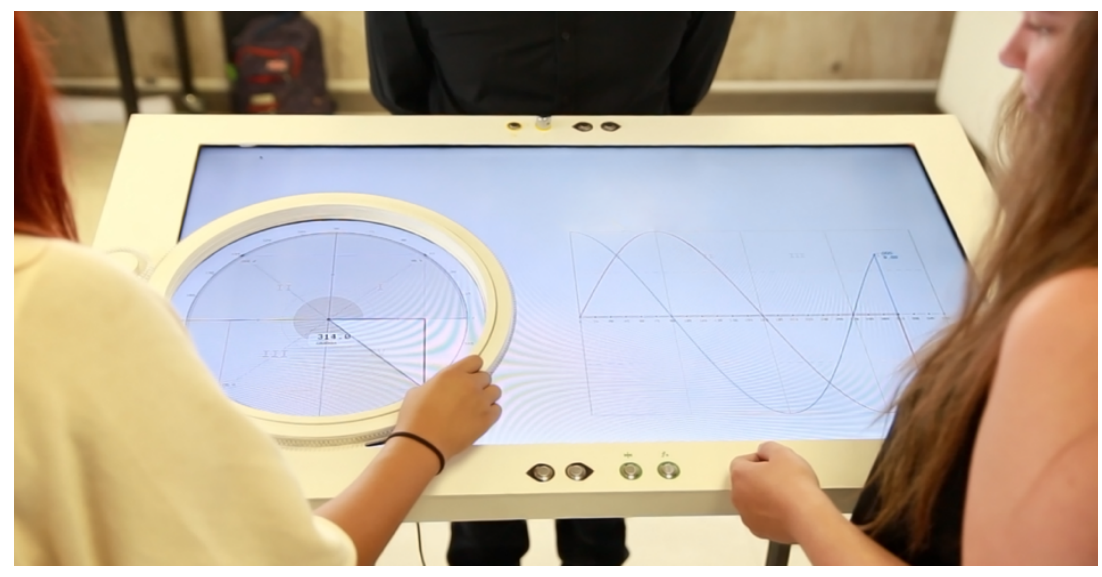

Fig. 1. Users manipulating the interface

\subsection{Pedagogical experience with the interface}

Five thematic modules were defined based on literature review and the previous experience of Engineering professors. The modules privilege the most introductory subjects to gain a basic understanding of how trigonometry works, focusing on the cartesian plane, the unit circle, sine and cosine, and the periodical nature of trigonometry. The five modules included:

- Module 1: Cartesian plane and polar coordinates

- Module 2: Sine values based on an angle

- Module 3: Cosine values based on an angle

- Module 4: Periodic functions and sine waves

- Module 5: Special angles and trigonometric identities

The pedagogical experience consists of a group of students and an instructor who interact with the interface during thirty-minute-long learning sessions. The instructoror "Facilitator"-guides students through the session, raising a series of questions about different concepts of trigonometry. These questions initiate the exploration through the manipulation of the interface and spark discussions within the group of participants (Figure 2). The progression of the experience is supported by a script structured in twelve sequential "scenes", each one including its own visual representation and corresponding activity. The script follows a constructivist approach, building 
up from the simplest to the most complex, scaffolding on the previous scenes to introduce new concepts through specific interactions. For instance, the first scene introduces the value of sine (represented by a red bar) on the $\mathrm{Y}$ axis of a cartesian plane. The length of the bar changes in response to the rotation of the Rotary Wheel. The second scene introduces the $\mathrm{X}$ axis along with cosine (represented by a blue bar) whose length is also modified using the Rotary Wheel. In the third scene, both axes and bars can be seen working together, providing the first approaches to the relationship between sine and cosine.

A very important aspect of the experience is that it uses a non-technical approach to the understanding of concepts. For instance, in the first quarter of the session the terms "sine" and "cosine" are intentionally replaced by "red bar" and "blue bar" until there is an understanding of how they work and where they come from before introducing the actual terms. In this way the experience enables participants to internalize the meaning of concepts before associating them with their name, facilitating students to incorporate the new knowledge with less resistance. This strategy bypasses the imprecise preconceptions that students might have about the concepts, which can distort or even prevent the acquisition of new knowledge [8].

The script encourages students to have clear spaces to err, experiment, explore and collaborate. It also provides guidance for the Facilitator in respect to the moment in which to ask the participants for specific interactions, what questions to ask, and determining the milestones that require an assessment of the understanding level. The underlying idea behind the script is that students are challenged with an open question and encouraged to work collaboratively on their own to solve it, promoting higher levels of thinking [32].

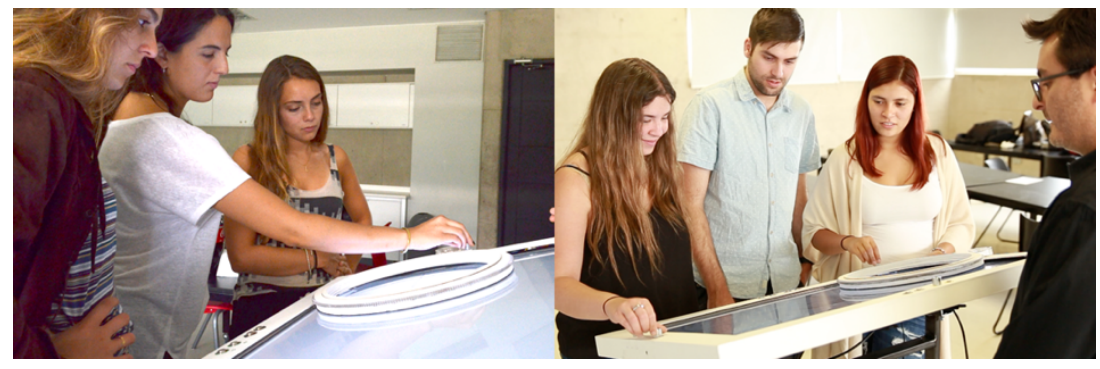

Fig. 2. Students and Facilitator interacting with the interface during sessions

\section{$5 \quad$ Methodology}

To evaluate the impact on learning of the proposed interface, 121 first-year students from the Design and Engineering schools participated in the study. Quantitative data was collected before and after an intervention with the interface. The intervention consisted in groups of students (3-6 per group) interacting with the interface. Each session lasted about 30 minutes and each student participated in two sessions during one academic year (ten months). 
A Pre-Test was applied at the beginning of the study (3rd month) to measure their initial knowledge, and a Post-Test was applied after the second session with the interface (7th month). Both tests were identical and included 12 questions, where each one was worth 1 point. The scope of the tests was limited to the basic conceptual knowledge (as defined in the Modules) and did not cover the application of trigonometry (i.e., problem-solving).

The Pre-Test was taken by 121 students, 66 from the Design School and 56 from the Engineering School. From that group, 24 students (10 from the Design School, and 14 from the Engineering School) participated as the Experimental Group. These students participated in the two sessions with the interface and took the Post-Test after the second session. A Control Group of 16 students from the Design School took both the Pre-Test and Post-Test without participating in any session with the interface and without receiving formal instruction (see Figure 3).

During the regular course of the academic year, Engineering students received trigonometry instruction in their Geometry course as defined in their curriculum. Design students did not receive any trigonometry instruction.

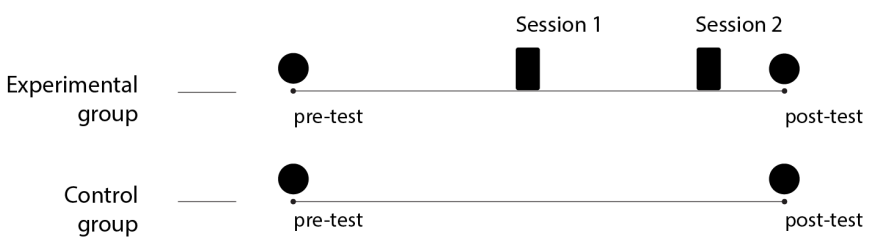

Fig. 3. User-testing and learning assessment activities for each group considered in the study

\section{$6 \quad$ Results}

\subsection{Pre-test results}

Results of the Pre-Test were low: as seen in Table 1, of a maximum of 12 total points, in Engineering the mean score was 4.35 points (36\% yield), and in Design of 2.44 points $(20 \%$ yield). Although the overall performance in Design was lower than in Engineering, when analyzing the results by thematic Module and by question, the trends were very similar in both careers, showing that the difficulties were coincident and that the level of understanding of basic concepts of trigonometry was transversally low (Figure 4).

Table 1. Mean scores and performance obtained by Design and Engineering students in the Pre-Test

\begin{tabular}{|l|c|c|}
\hline \multicolumn{1}{|c|}{ Career } & Mean Score (12 points max.) & Performance \\
\hline Design $(\mathrm{n}=65)$ & 2.44 & $20 \%$ \\
\hline Engineering $(\mathrm{n}=54)$ & 4.35 & $36 \%$ \\
\hline
\end{tabular}



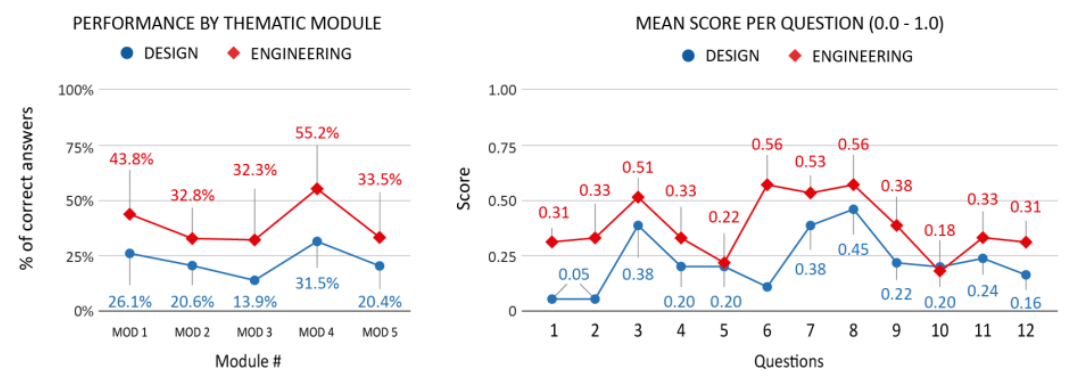

Fig. 4. Pre-Test results by Thematic module (left) and by question (right). As observed, the performance curves are similar in both careers.

\subsection{Comparative results}

When comparing the results of the Pre and Post-Test, a significant improvement was observed in the Experimental Group (statistically confirmed by a two-sample ttest and a paired sample t-test). Figure 5 shows that in Design, the mean increase was of 5.0 points, and in Engineering 3.9 (4.46 points when considering both careers). It is noteworthy that even though Engineering students were exposed to additional formal instruction in trigonometry during the lapse of this study (as opposed to Design students) their score improvement was comparatively lower than the score improvement of Design students. Further studies are needed to assess with greater detail the influence of the interface in the case of students receiving additional trigonometry instruction, but the current analysis supports that using the interface had an impact on students' performance overall.

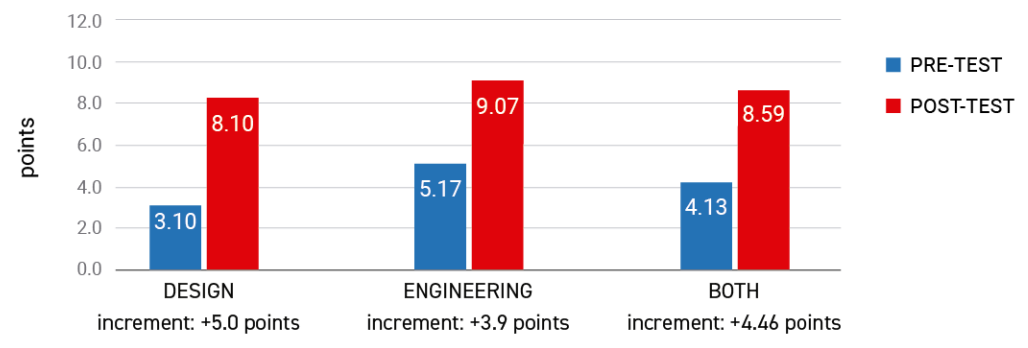

Fig. 5. Comparison of Pre-Test and Post-Test results in the Experimental Group

Table 2 shows the comparative performance per question in the Pre-Test and PostTest for the Experimental Group as a whole. After going through the experience with the interface, performance increased from $34.4 \%$ to $71.6 \%$, yielding a differential of $+37.1 \%$, which indicates that the experience positively impacted learning. 
Table 2. Performance per question on the Pre-Test and Post-Test, including both schools

\begin{tabular}{|c|c|c|c|}
\hline Question & Pre-Test performance (\%) & Post-Test performance (\%) & Differential (\%) \\
\hline 1 & 12.5 & 91.4 & +78.9 \\
\hline 2 & 17.5 & 91.4 & +73.9 \\
\hline 3 & 39.2 & 96.4 & +57.3 \\
\hline 4 & 45.0 & 92.9 & +47.9 \\
\hline 5 & 27.5 & 47.1 & +19.6 \\
\hline 6 & 35.0 & 77.9 & +42.9 \\
\hline 7 & 62.5 & 77.9 & +15.4 \\
\hline 8 & 61.7 & 78.6 & +16.9 \\
\hline 9 & 40.0 & 38.6 & -1.4 \\
\hline 10 & 18.3 & 65.7 & +47.4 \\
\hline 11 & 30.8 & 70.7 & +39.9 \\
\hline 12 & 23.3 & 30.0 & +6.7 \\
\hline Average & $34.4 \%$ & $71.6 \%$ & $+37.1 \%$ \\
\hline
\end{tabular}

The results of the Design students within the Experimental Group $(n=10)$ are particularly noteworthy, showing an increase of +5.0 points, as opposed to the Control Group $(n=16)$ that only increased +0.64 points (Figure 6$)$. This difference strengthens the conclusion that the performance increase is mostly attributable to the interface, and that being exposed to the same test twice did not have a relevant impact on their scores.

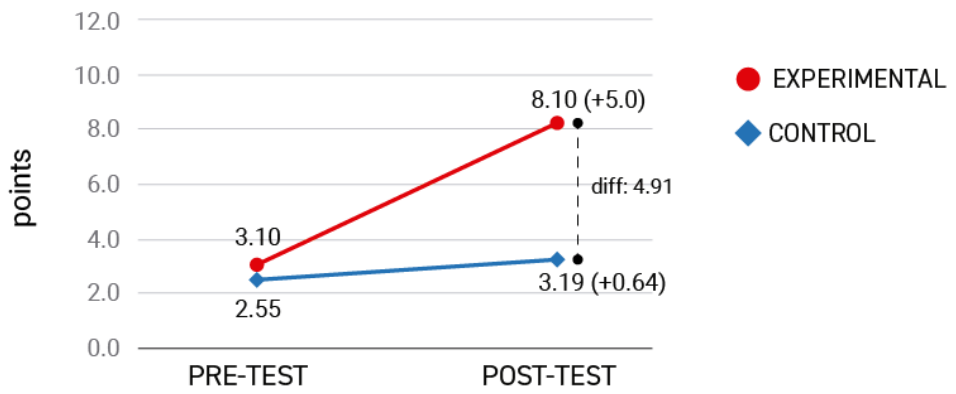

Fig. 6. Scores obtained in Pre-Test and Post-Test by the Experimental and Control groups from the Design School. It can be observed that the Control Group does not show a significant improvement, whereas those exposed to the interface increased their score by +5.0 points.

\section{Discussion and Conclusion}

The present study was developed based on the detected potential and incipient use of TUIs to enhance the learning process of abstract domains [33]. Specifically, we focused on the difficulties evidenced in the teaching-learning of trigonometry in secondary and higher education and explored the possibilities of improving conceptual 
understanding by interacting with a TUI. Although there are effective software-based tools that include trigonometric content such as Geogebra [10], they are constrained to single-user operation and do not incorporate collaborative learning through the use of tangible components.

In the case of this study, the designed interface hosts a pedagogical experience to learn trigonometry that privileges bodily interactions through exploration and physical manipulation [22] to foster intuitive, playful [18] and collaborative learning. The purpose is to explore abstract mathematical concepts through tangibility-applying principles of the theory of embodied cognition [15]. This produces significative learning by resorting to the knowledge acquired by manipulating daily objects.

Participants reported that using multiple senses to explore contributed to diversify their access routes to information during the learning experience. By observing users interact with the interface, we confirmed that being able to "touch and move" representations of digital information proved to be beneficial to internalize abstract concepts in concrete terms [15]. For instance, rotating the Rotary Wheel and seeing how that changes the angle, sine and cosine values in real-time, is one of the ways in which a natural gesture helps to make the abstract become concrete. The use of sound was particularly well received by participants, because it helped them to focus on relevant aspects in key moments and enabled an additional representation in an innovative and unexpected way.

The pedagogical experience was designed based on a constructivist approach, using the interface as a mean to encourage students to access and question their prior knowledge [8] by combining it with new information coming from the interface and their peers [9]. One of the key differences offered by the interface-as opposed to traditional teaching styles-is the freedom to err and to navigate in a non-linear way. The "learning by doing" approach [12, 27] encouraged experimentation and acceptance of "failure" within the participants. In addition, the continuous validation of acquired knowledge during the session enabled students to feel empowered to incorporate more complex concepts progressively.

Students self-reported that the experience positively influenced their comprehension of trigonometry, helped them clear misconceptions, and learn collaboratively. The script was developed to provoke these interactions by posing a problem and encouraging students to work on their own to solve it, with the aim to promote higher levels of thinking and self-discovery. It was inspiring to observe the process of cocreation of knowledge among participants from different disciplines when they discussed issues while manipulating the interface. They had to reflect and agree on opinions, thus making the learning process more dynamic and participatory.

The design of the pedagogical experience resulted in dynamic and fun sessions, which were positively evaluated by participants. The style used by the Facilitator played an important role: using a simple and non-technical language during the experience helped students to feel at ease with a subject that was initially perceived as difficult and distant. This informal atmosphere promoted the construction of metaphors that served to achieve an understanding of abstract concepts [34].

The designed interface endorses the potential of TUIs as pedagogical tools to facilitate trigonometry learning. Quantitative results demonstrate that students exposed 
to the interface effectively enhanced their understanding of basic trigonometry concepts and improved their measured performance. By focusing first on the understanding of the concepts instead of on the direct resolution of mathematical problems, the research team believes that the interface has the potential to become a useful ally for educators to facilitate the transition to the more abstract domains needed for advanced mathematics.

\section{Acknowledgements}

This research is supported by the Office of Research and PhD at Universidad del Desarrollo. Further support was provided by the Design Research Society. We want to thank our research assistants María Elena Errázuriz and Joaquín Domínguez for their important role in the development of the interface, and to Francisco Fuentes for the collaboration on the ideation process. We also want to thank Viviana Solano from the School of Engineering for her support and management with students. Finally, we want to thank all the participants who tested and provided feedback during the design process.

\section{$9 \quad$ References}

[1] Curri, E. (2012). Using Computer Technology in Teaching and Learning Mathematics in an Albanian Upper Secondary School: The Implementation of SimReal in Trigonometry Lessons. Universitetet i Agder; University of Agder.

[2] Weber, K. (2005). Students' understanding of trigonometric functions. Mathematics Education Research Journal, 17(3), 91-112. doi:10.1007/BF03217423

[3] Brown, S. A. (2006). The trigonometric connection: Students' understanding of sine and cosine. Proceedings of 30th Conference of the International Group for the Psychology of Mathematics Education, vol. 1 (Vol. IV).

[4] Thompson, P. W., Carlson, M. P., \& Silverman, J. (2007). The design of tasks in support of teachers' development of coherent mathematical meanings. Journal of Mathematics Teacher Education, 10(4-6), 415-432. https://doi.org/10.1007/s10857-007-9054-8

[5] Skinner, B. F. (1976). About Behaviorism. Vintage.

[6] Johnson, M. (2013). The body in the mind: The bodily basis of meaning, imagination, and reason. University of Chicago Press.

[7] Méndez, W., \& Leal, S. (2018). Mathematical modeling and application problems as promoters of creativity in the teaching and learning of trigonometry. Revista de Investigación, 42, 136-157. Retrieved from http://www.upel.edu.ve

[8] Ambrose, S., Bridges, M., Dipietro, M., Lovett, M., \& Norman, M. (2010). Seven Research-Based Principles for Smart Teaching (Vol. 48). doi:10.1002/mop.21454

[9] Vygotsky, L. S. (1980). Mind in society: The development of higher psychological processes. Harvard university press.

[10] Dockendorff, M., \& Solar, H. (2018). ICT integration in mathematics initial teacher training and its impact on visualization: the case of GeoGebra. International Journal of Mathematical Education in Science and Technology, 49(1), 66-84. https://doi.org/10.1080/00207 $\underline{39 x .2017 .1341060}$ 
[11] Velichová, D. (2011). Interactive maths with GeoGebra. International Journal of Emerging Technologies in Learning, 6(SPECIAL ISSUE.2), 31-35. https://doi.org/10.3991/ijet.v6is $\underline{1.1620}$

[12] Kepceoğlu, I., \& Yavuz, I. (2016). Teaching a concept with GeoGebra: Periodicity of trigonometric functions. Educational Research and Reviews, 11(8), 573-581. https://doi.org/ $\underline{10.5897 / \text { err2016.2701 }}$

[13] Mushipe, M., \& Ogbonnaya, U. I. (2019). Geogebra and Grade 9 Learners' Achievement in Linear Functions. International Journal of Emerging Technologies in Learning (iJET), 14(08), 206-219. https://doi.org/10.3991/ijet.v14i08.9581

[14] Lakoff, G. (2009). The Neural Theory of Metaphor. Ssrn. doi:10.2139/ssrn.1437794

[15] Lakoff, G., \& Núñez, R. E. (2000). Where Mathematics Comes From: How the Embodied Mind Brings Mathematics into Being (1st ed.). New York, NY, USA: Basic Books. https:// doi.org/10.2307/3072449

[16] Ishii, H. (2007). Tangible User Interfaces. In Human-Computer Interaction: Design Issues, Solutions, and Applications (pp. 141-157). CRC Press. https://doi.org/10.1201/97814200 88861.ch8

[17] Ishii, H., \& Ullmer, B. (1997). Tangible Bits: Towards Seamless Interfaces between People, Bits and Atoms. PAPERS CHI (Vol. 97). https://doi.org/10.1145/258549.258715

[18] Schneider, B., Jermann, P., Zufferey, G., \& Dillenbourg, P. (2011). Benefits of a tangible interface for collaborative learning and interaction. IEEE Transactions on Learning Technologies, 4(3), 222-232. https://doi.org/10.1109/tlt.2010.36

[19] Starčič, A. I., Turk, Ž., \& Zajc, M. (2015). Transforming pedagogical approaches using tangible user interface enabled computer assisted learning. International Journal of Emerging Technologies in Learning (iJET), 10(6), 42-52. https://doi.org/10.3991/ijet.v10i6.4865

[20] Marshall, P., Price, S., \& Rogers, Y. (2003). Conceptualising tangibles to support learning. In Proceeding of the 2003 conference on Interaction design and children - IDC '03 (p. 101). New York, New York, USA: ACM Press. https://doi.org/10.1145/953536.953551

[21] Antle, A. N., \& Wise, A. F. (2013). Getting Down to Details: Using Theories of Cognition and Learning to Inform Tangible User Interface Design. Interacting with Computers, 25(1), 1-20. https://doi.org/10.1093/iwc/iws007

[22] Resnick, M., Myers, B., Nakakoji, K., Shneiderman, B., Pausch, R., Selker, T., \& Eisenberg, M. (2005). Design Principles for Tools to Support Creative Thinking. NSF Workshop Report on Creativity Support Tools, (Creativity Support Tools), 25-35. https://doi. org/10.1207/s15327590ijhc2002_1

[23] Dodge, E., \& Lakoff, G. (2005). Image schemas: From linguistic analysis to neural grounding. From perception to meaning: Image schemas in cognitive linguistics, 57-91. https://doi.org/10.1515/9783110197532.1.57

[24] Feldman, J. (2008). From molecule to metaphor: A neural theory of language. MIT press.

[25] Pecher, D., Boot, I., \& Van Dantzig, S. (2011). Abstract Concepts: Sensory-Motor Grounding, Metaphors, and Beyond. Psychology of Learning and Motivation, 54, $217-$ 248. https://doi.org/10.1016/b978-0-12-385527-5.00007-3

[26] Camilleri, M. A., \& Camilleri, A. C. (2017). Digital Learning Resources and Ubiquitous Technologies in Education. Technology, Knowledge and Learning, 22(1), 65-82. https://doi.org/10.1007/s10758-016-9287-7

[27] Zengin, Y. (2018). Incorporating the dynamic mathematics software GeoGebra into a history of mathematics course. International Journal of Mathematical Education in Science and Technology, 49(7), 1083-1098. https://doi.org/10.1080/0020739x.2018.1431850

[28] De Raffaele, C., Smith, S., \& Gemikonakli, O. (2018). An Active Tangible User Interface Framework for Teaching and Learning Artificial Intelligence. In Proceedings of the 2018 
Conference on Human Information Interaction\&Retrieval - IUI '18 (pp. 535-546). New York, New York, USA: ACM Press. https://doi.org/10.1145/3172944.3172976

[29] Kendal, M., \& Stacey, K. (1996). Trigonometry : Comparing Ratio and Unit Circle Methods University of Melbourne , Australia. In Technology in Mathematics Education. Proceedings of the 19th Annual Conference of the Mathematics education Research group of Australasia (pp. 322-329).

[30] Mesa, V., \& Goldstein, B. (2016). Conceptions of Angles, Trigonometric Functions, and Inverse Trigonometric Functions in College Textbooks. International Journal of Research in Undergraduate Mathematics Education, 3(2), 338-354. https://doi.org/10.1007/s40753016-0042-1

[31] Shams, L., \& Seitz, A. R. (2008). Benefits of multisensory learning. Trends in Cognitive Sciences, 12(11), 411-417. https://doi.org/10.1016/j.tics.2008.07.006

[32] Gilbert, N. J., \& Driscoll, M. P. (2002). Collaborative knowledge building: A case study. Educational Technology Research and Development, 50(1), 59-79. doi:10.1007/BF02504961

[33] De Raffaele, C., Smith, S., \& Gemikonakli, O. (2018). An Active Tangible User Interface Framework for Teaching and Learning Artificial Intelligence. In Proceedings of the 2018 Conference on Human Information Interaction\&Retrieval - IUI '18 (pp. 535-546). New York, New York, USA: ACM Press. https://doi.org/10.1145/3172944.3172976

[34] Font, V., Bolite, J., \& Acevedo, J. (2010). Metaphors in mathematics classrooms: Analyzing the dynamic process of teaching and learning of graph functions. Educational Studies in Mathematics, 75(2), 131-152. https://doi.org/10.1007/s10649-010-9247-4

\section{Authors}

Francisco Zamorano Urrutia is an instructor and researcher at the Design School at Universidad del Desarrollo. He holds a Master of Fine Arts in Design \& Technology from Parsons School of Design, NY, United States. His work explores the intersection between education, novel interfaces, motion design and experiential design.

Catalina Cortés Loyola is an Instructor and Researcher of the Design School at Universidad del Desarrollo. She holds a Master of Science in Design from Arizona State University, United States. Her areas of interest are: learning and cognition, design literacies for the general public, design of educational resources and learning environments.

Mauricio Herrera Marín is an Instructor and Researcher of the Engineering School at Universidad del Desarrollo. He holds a Post-doctorate from the Institute Weizmann of Science, Rehovot, Israel and a Ph.D. in Physics from Universidad de Chile.

Article submitted 2019-08-02. Resubmitted 2019-09-25. Final acceptance 2019-09-26. Final version published as submitted by the authors. 